\title{
A Review of Secure Authentication based e-Payment Protocol
}

\author{
Mr.B.Ratnakanth \\ Dep.of Computer Science and Systems Engineering \\ Andhra University \\ Visakhapatnam, India
}

\author{
Prof.P.S.Avadhani \\ Dep.of Computer Science and Systems Engineering \\ Andhra University \\ Visakhapatnam, India
}

\begin{abstract}
The growth of e-commerce platform is increasing rapidly and possesses a higher level of hazard compared to standard applications as well as it requires a more prominent level of safety.Additionally, the transaction and their data about clients are enormously sensitive, security production and privacy is exceptionally crucial. Consequently, the confirmation is generally vital towards security necessities as well as prevents the data from stolen and unauthorized person over the transaction of e-payment. At the same time, privacy strategies are essential to address the client data security. Because of this, the data protection and security ought to be viewed as a central part of ebusiness framework plan. Particularly enormous consideration is given to cash exchanges assurance. In the past decades, various methods were created to permit secure cash transaction using epayment frameworks. This study will review and discuss the epayment scheme. It uses various encryption algorithms and methods to accomplish data integrity, privacy, non-repudiation and authentication.
\end{abstract}

Keywords-Security protocol; smart card; encryption technique; payment protocol; E-commerce

\section{INTRODUCTION}

The internet is raised the area for social communication as well as coordinated effort. Specifically, it chose source to online accessible e-services like e-banking, e-voting, egovernment, e-commerce and so on. it permits organization as well as persons towards exchange ideas, communicate, services and trade goods as more productively [1]. In the developing countries, the internet has been warmly accepted and established for social interaction with information. However, the part of the internet in e-commerce (commerce/trade) is still extremely constrained. The ecommerce plays an essential part in financial improvement through decreasing the expense of services and products. Also, it is known as conducting business through online. Further, it has a capable to offer sell or buy the products, services, and information through online as well as used for other internet applications. The amount transaction is necessary for various trading action, and it should be reliable and safe between transaction parties. The e-payment method is the essential part in e-commerce platform[2]. The EPSs (Electronic payment system) enables the significant role afterward the client choice towards paying the services or products as well as carry payments from client to sellers in an effective way[3].Additionally, the requirement of payment through mobile phone have emerged for the growth of mobile electronic commerce [4]. Also, the EPSs system encourages medium and small size enterprises as well as permits them towards contend with giants in the same commercial center [5]. The web application wants a robust security that has a prominent attribute towards ensuring client secret information. Additionally, on the internet based e-payment framework security is the main problem. There are various web dangers that increase the risk as well as influence the security scheme on behalf of the electronic transaction. Mostly, this depends on individually recognizable proof numbers, keys to get to their own record data and passwords. This sort of validation framework can't confirm or verify the personality of the clients who he/she rights to be[6]. Over an insecure communication channel, the security of e-payment is a challenging task which incorporates numerous serious zones as a robust encryption method, trusted third party and protected communication channel towards maintaining the online database[7].

\section{A. Need for the Study}

The internet is the communication channel that permits more people or organizations towards converse each other without abundant efforts and charges. Recently the online hacker is widespread all over the world, and it makes main resource profits for criminals. Presently online misrepresentation is extremely famous everywhere throughout the world; it has turned into a noteworthy wellspring of income for offenders. The banks or budgetary foundations are extremely mindful in identifying and counteracting online fakes [8], [9].In spite of that electronic business is a developing marvel that upcoming improvement towards a substantial range, vulnerable through the absence of proper payment framework. Subsequently, the majority of the business to customer installments through internet recently performed by MasterCard's and indeed problematically payment medium because of security, trust issues, and cost. Further, the requirement of new payment scheme obviously rises up out of the previous circumstance [10]-[12]. In order to resolve this situation, previous research, and development in internet based payment system proposed by online based epayment system, a great extent of which has been put to utilize. This was conceivable because of the fortifying variables scheduled above, and in any case because of the reducing cost and availability of the assisting technology[13]. For overcoming these issues in the e-payment field, need to propose a conventional payment system for online business using an intermediate. 


\section{RELATED WORK}

Recently, the secure wireless transactionschemehas turned into a research extent towards payment system.Some of them have concentrated towards security and privacy issues alone such as Ray et al. [14], Kouta et al. [15], Cui et al. [16], Tiwari et al. [17],Raghuwanshi et al. [18], Abdellaoui and Pasquet[19], Mazumdar and Giri[20], Takyi and Gyaase[21],Lawal et al. [22], Khan et al. [5],Aigbe and Akpojaro[23],Kim et al. [24]. On the other hand, focused on secure mobile payment system[25]-[30]and some of them have focused Secure Electronic Transaction protocol[31][33].Those are discussed as follows.

\section{A. Studies Related to Secure Mobile Payment System}

$\mathrm{Hu}$ et al.[34]proposed a secure mobile micropayments using AMA method. By this method, the customer gets a services or goods from mercantile in various domains through mobile micropayments except disclosing his/her privacy. Additionally, without expanding correspondence overheads noticeable all around, the most computational exertion is moved to the wired system towards reducing the computational overheads on a smart card or mobile with restricted storage and computational capacity. Further, the execution of bottlenecks of the whole framework focuses the clearing, credential, and settlement.Subsequently, sometimes the credential center is also difficult issues in this payment system. In the cryptography based symmetric key approach, this is a common issue but in the future studies, this would be improved.

Tabandehjooy and Nazhand [25]proposed a secure and lightweight protocol for mobile payments. Further, they prevent their non-repudiation as well as authenticate a client using the complex key. In this cryptography method, each data exchange the information, and it makes integrity and more confidentiality. Also, it improves the trust payment. The results show that the protocol considerably improves the customer trust through complex key since not ever enter their secure data in the electronic payment system.

Sazzad et al.[26]proposedmobile banking system using SMS services and offered an opportunity to all customer of economic foundation towards utilizing the available facilities through netbanking. This scheme mainly used for all users and does nor require any refined internet connectivity or cellular phone in the mobile handset.Also, they provided main services such as amount transfer between authorized clients, balance inquiry, bill payment as well as save the precious time. The internet base secures communication between banking and mobile server that was beyond the client control and is handled critical problems. Also, employed audio based digital watermarking for providing higher security with voice authentication. By this secure handle system, the clients easily attract to e-banking, specifically in emerging countries.

Suryotrisongko and Setiawan[35] proposed a mobile payment scheme for the cooperative system. To improve the security, they used quick response encrypted message and two-factor authentication. However, this study needs to be validated and tested most thoroughly in security aspect. Furthermore, more massive experiments and survey need to be conducted in order to measure user's acceptance regarding this model.

Mwafise and Stapleton[36]discussed the institutional and socio-technical domain to attain technology adaptation. However, this study was restricted in that it didn't exploit results in different locales which will have been a rich contribution to either reinforcing a portion of the focuses created or in testing provincial contrasts of perspectives on innovation selection which could likewise be helpful in building up a strong model of the deterministic variables on innovation acceptance in emerging countries.

Isaac and Zeadally[27]put forth a proposal for a protocol that was lightweight in order to achieve on-line payments that were secure. This was in a limited eventuality where a direct mode of communication could be possible between the clients and merchant. The protocols that postulate rely on symmetric cryptographic techniques which function effectively on lower requirements of computation. Additionally, the Payment Gateway plays an active role in processing payments as it discharges and its role as a proxy that permits communication between the client and merchant. Even though the suggested protocol was devised for a mobile payment system for a limited eventuality, the security properties remain intact. The study of the performance indicates that the mobile payment protocol that is suggested demands lesser computation than that required for KSL and LMPP, which in turn results enhanced end-to-end performance and can be installed on mobile devices operating with minimum or reduced computational resources.

Kim et al. [28]studied the security threats, requirements of security, and various security modules as a user processes the payment through NFC-based mobile. Mobile payment offers convenience and efficiency. But, at this situation, the process of payment is initiated post-authentication by validating key information, such as card and personal information which is stored and handled on NFC-based USIM. Therefore, for the purpose of secure mobile payment, the components design and modules was crucial. Hence, in this study, for a secure mobile payment system through NFC, examined the threats to security, the requirements and the requirement of every module in payment procedures. This will undertake a study in the future about the protocol that devised and utilized superior and strong encryption algorithms as well as safe, secure validation mechanisms that can tackle security threats.

Ahamad et al. [29]suggested a Secure Mobile Payment Framework (SMPB) that relied on biometrics and worked through Universal Integrated Circuit Card (UICC) and Wireless Public Key Infrastructure (WPKI). Additionally, they carried out a comparative analysis between this method and works in the recent past and learned that this method was a better option in the context of achieving an end to end security.This mobile payment protocol which originates from Mobile Payment Application to Bank Server results in Fair Exchange, ensuring Authentication, Confidentiality, NonRepudiation, and Integrity, pre-empts spending twice, spending above limits and money laundering, in addition, to being able to remain resilient duringreplay, MITM (Man in the Middle) and Impersonation attacks. A plan exists to validate 
the mobile payment protocol by utilizing AVISPA and Scyther Tool in the anticipated circumstances.

Lomte et al. [30]put forth a proposal for a secure payment protocol, taking into consideration the limitations of cellular networks in developing nations. Moreover, this method fulfills the satisfaction quotient in terms of convenience and ease of use, two criteria of mobile users making small transactions. Additionally, it offers security for the transaction and nonrepudiation property which is mandatory for macro payments. Even though the suggested technique was developed so as to be in harmony with the present GSM network, the modular design envisaged is future-ready, it will accept improvements in the future to mobile network technology and associated infrastructure, for instance, EMS and MMS, requiring minimal changes to the protocol structure. But, the exact nature of implementation of workflow will ultimately depend on the disposition of the user. Like a light motive, businesses with multichannel infrastructure need to unify harmoniously the level of security for m-payment and security architectures that are web-based for m-payment so as to safeguard their businesses and for the development of future-proof architectures.

Ting et al. [37]investigated the effects of subjective norm, attitude with perceived behavioral control on intention by mobile payment system amongst Chinese and Malays in Malaysia, through the use of underlying based planned behavior theory. They collected and tested the data by independent sample t-test with multiple linear regressions in SPSS. Because of the limits of SPSS, four separate models are needed towards executing the regressions. Furthermore, when performing bunch examination by ethnicity, the extension is delimited to Malays and Chinese. Thus, future studies can be directed utilizing Structural Equation Modeling (SEM) to better clarify expectation towards m-installment framework in a solitary auxiliary model.

\section{B. Secure Electronic Transaction Protocol}

Guan et al. [31]put forth an expandable SAFER (Secure Agent Fabrication, Evolution \& Roaming)-based e-payment module that meets the requirements of commerce depending on agents. Secure Electronic Transaction (SET) and E-Cash protocols were selected to function as modes of payment. The well-defined interface also makes possible the inclusion of additional features in one single module, without affecting the reliability in other related modules. Further improvements in future of the system may see the inclusion of agent security measures. Additional research has been undertaken in this field by projects running paralleled, and the results obtained can be utilized to improve the present system. Additionally, different electronic payment schemes can be considered for implementation in the form of additional payment modules so as to build on the flexibility of this framework as well as convenient to users.

Wang and Varadharajan[32]posit a secure payment protocol that was agent-assisted in a manner that supports multiple payments, which utilizes Signcryption-Share scheme and Signature-Share scheme employing a Trusted Third Party (TTP). The protocol that has been suggested put forth the principle that every player with a role is aware of what is actually mandatory for him/her and followed similar to SET when the non-repudiation property gets improved. The dispatch agents selects flexibly and in a dynamic manner the merchant and affix a sign for the cardholder with the consent of the TTP, and all of this executed without having to reveal any information of a secret nature pertaining to the card to the merchants and the TTP. The information of offers is safeguarded from unrelated merchants. In order to minimize the risk of using the services of mobile agents, the reliability and integrity of merchants can be assessed well in advance. Nevertheless, this paper has to coordinate the suggested protocol into the PumaMart system for the purpose of enhancing performance $-\mathrm{a} \mathrm{B} 2 \mathrm{C}$ marketplace that is mediated by an agent [38], [39] executed over and above Java and IBM Aglets toolkits [40].

Sun [41]SET protocol is a system that is concentrated, comprising a request for the request, a payment verification, and the final payment through means. To begin with, the trade process is categorized as per the various transaction amounts, followed by the optimization of the lightweight transaction process, and testing of the SPIN model of theSET protocol.Eventually, the SET protocol is enhanced as per the outcome of simulation and testing.Addition, validation of the security protocols that select the encryption algorithm and the protocol agreement in combination with the encryption technology to ascertain the presence of the attacker at an increased agreement level.

Ismaili et al. [33]suggested a three faceted (3D) system of security, which includes 3-D Secure and 3D as methods of enhancing the security of e-commerce transaction. On the fundamentals of SSL, SET, 3D security schemes and the specific needs of electronic payment, a safe, secure and effective E-Payment protocol has been devised.This presents an additional level of protection for merchants and cardholders. Customers are requested to key in a separate password after the completion of checkout to ascertain if the person is indeed the legitimate card holder; the validation is carried out directly between the card issuer and cardholderutilizing the security certificate apart from the act of not utilizing the third party (Visa, Master Card). Nevertheless, this paper needs to treat analysis of security and performance as the lynchpin of the suggested protocol. Sfenrianto [42] examined the client intention towards use e-payment scheme. However, they specifically focused in Indonesia.

\section{Studies Related to Smart Card}

Mobahat [43]debated various protocols regarding validation and cryptography in low-cost RFID and carried out a comparison of the resultant output with a qualitative approach. This plan ensures that there is a chance to unearth similitudes and variations among protocols and corresponding solutions. This permits specialists or executives on the look out for a proper protocol or method that matches their requirements and priorities, could select one out of two among many; to put it in a different way, while investigating various types of attacks launched against schemes, either the administrator or customer using the technology could establish or refer to a comparison to determine the appropriate protocol as per the criteria or priorities; such as the value of data transmitted by RFID transceivers located in airfields or 
wireless media, or the potential attacks that could occur as per the location of RFID devices. Nevertheless, during the course of this paper certain issues could not be explored, i.e. the privacy and security properties. However as per the references, the same could be investigated and considered in works and researches in the future.

Madhoun et al. [44]brought out a novel security protocol for payments and transactions through NFC which resolved the security vulnerabilities which were identified in the EMV protocol. This is based on cloud infrastructure to ascertain the authenticity of payment terminals and offer confirmation to smartphones. This ensures mutual verification, nonrepudiation among NFC smartphone, NFC payment terminals, reliability, and the maintenance of confidentiality of information of private banking. They successfully examined in depth the accuracy of the protocol through the Scyther authentication tool that offers standardized proof to verify security protocols. They intend to offer a better solution, create a prototype and display its efficiency in in a real scenario.

Pal et al. [45]suggested a model which involves two client oriented features with four system oriented features in general. Further, they empirically evaluated this study and segmented the clients into two groups such as late and early adapters. Also found the features which affect the client intention to this system. The experimental solution shows this approach apparent helpfulness is the two robust interpreters for clients acceptance of NFC-based mobile payment scheme and ease of use. However, this payment system, the security problems with government support were not considered. Additionally, for every sample which took mostly use debit or credit card. In any case, this can turn out to be an inclination component as it is not known how individuals not utilizing such cards will respond to this new framework.

\section{Studies Related To Security, Privacy, And Encryption Based Protocol}

Ray et al. [14]suggested a protocol that could be termed sanguine wherein the trusted third party is used only when any partyconducts itself in a manner that is inappropriate or aborts prematurely.By utilizing this protocol, a reasonable amount of fairplay is achieved and disputes are resolved in an automatic manner within the boundaries of protocol. This additionally demonstrates the manner in which the function of the third party is spread out across many third parties; this improves the robust nature of the protocol. Additionally, this also indicates the manner in which a payment mechanism needs to be adopted; transacted electronically, offered discretion to the transaction of customers. Moreover, they intended to execute such systems in the future. In order to examine the protocol through conventional software specification and authentication tools such as FDR etc. Specifically, they intend to examine the input by the trusted third party and determine the nature in which the frequency of failure by the third party has a bearing or influence on the performance. This study will assist in identifying methods to manage the protocol in the most effective manner. Finally; they intend to execute the protocol. The intention is to rely on COTS components for implementing. Execution will offer us a divergent view on protocol and may necessitate addressing of new issues.

Koutaet al. [15]discussed various methods using multiple agents which have implemented towards offering security as well as proposed agent-based scheme for e-payment. This approach has a substantial overhead that was initiated through multiple agents. It needs there is no interface between the inventor when the mobile agents as send out. Additionally, this proposed scheme resolve the security threats that prime towards new notes through threshold signature method in mobile agents.

Cui et al. [16]designed a typical E-voting scheme relying on a blind signature or discrete channel of communication channel - it is difficult for them to resolve fresh issues such as a vacant ballot, fraud or cheating, vote collusion, etc. The list signature is an extension of group signature, which includes public detection. Therefore, the suggested scheme can fulfill the aspects of E-voting and will resolve fresh problems in a convenient manner. In comparison with the conventional schemes, it is easier to locate members who give more than a single ballot in the suggested scheme. The results of the experiment display the safety and performance of this method.

Tiwari et al. [17]suggested a solution that uses applicationlayer security for a wireless payment system that offers endto-end verification and protection of data among wirelesses J2ME based clients and J2EE based servers. This study proposes a novel protocol for verification of web users based on multifactor authentication approach which is proven to be completely safe and convenient to execute. Also, propose a method for two-way verification protocol to validate both parties. This solution can effectively be executed within the controlled resources of a Java MIDP device, without the need for modifications to the fundamental protocols or wireless network infrastructure. They intend to concentrate on devising a novel and effective method to obtain TIC codes from financial organizations. TIC code installation on the cell phone of the users should also necessarily be a simple task to prevent frequent visits by a user to the bank or financial organizations. Server side TIC maintenance and a mechanism to manage it so as to fulfill the requirements of numerous users should also be put down as necessary work to be undertaken in the future.

Raghuwanshi et al. [18]suggested mathematical model towards validating the integrity of payment method as well as ordered details through online. This was based on the third party confirmation which receipts different messages from merchant and client. Also, integrity was verified. This scheme has the low-cost implementation and simple to use. However, this approach has involved the appraisement of the floating point. Furthermore, it is related to the integer computation or improves the efficiency as well as accurateness of calculation.

Abdellaoui and Pasquet[19]suggested a novel creation that will present itself in electronic payment transactions and termed as a payment service provider; it executes payment interactions for customers and merchants on the part of the private banking network. This novel payment scheme takes 
care of the issues of trust and safety. In order to handle this problem ofpayment, the service provider needs to suggest a safe and foolproof solution that will be convenient to coordinate with the web application of the merchant and ensure that the client has a good experience. But, this paper has not concentrated on the methods to integrate the three components or entities in the payment method that includes all other parties - it will be of interest particularly in an innovative payment platform that relies on the combination of many modes of payment (such as credit card, gift card) and the new challenges faced by e-commerce.

Mazumdar and Giri [20]proposed encryption approach for the design of secure protocol using online e- payment system. The token message was rationalized through distributing bank. Further, they verified the sender and user payment details as well as consent of client and mercantile. It offers truthfulness, fairness, secrecy and confidentiality.

Takyi and Gyaase[21]suggested that Robust Electronic Payment Protocol (REPP) fares better when compared theoretically with live cardholder verification in terms of security, usability, verification of cardholder and execution. But, the REPP authenticates the merchant just as SET does. Hence, the results prove that REPP has a better capability to reduce the chances of fraud, easy to use, and convenient to execute in a real world scenario. This suggested protocol could serve as the perfect antithesis to defrauding activities that are being witnessed in e-commerce markets. But, they were debated only with reference to theoretical issues. Additionally, theprotocol will be executed to analyze and prove its strengths.

Lawal et al. [22]discussed Multifactor Authentication approaches for e-payment and banking services of banks. Similarly, assessed the various kind of security scheme as well as a developed measure of security to verify the client retrieved their financial services using online. Also, address the customer awareness program as well as risk-based evaluations were conducted. However, they only focused on marketable banks in Nigeria. Also need to focus towards evaluates the efficiency and reliability of the system using 2factor authentication.

Khan et al. [5]present the potential for development of ecommerce, analyze it with a fresh protocol termed as "DualNetwork E-Payments Protocol" which was suggested in Pakistan. The protocol relies on a grouping of GSM and IP networks. It fulfills all the desired characteristics of an epayment mechanism. The protocol is reasonably safe and secure, reliable and appropriate for developing local infrastructure. Dual Network e-Payments Protocol relies on internet \& GSM networks. This protocol is devised on the basis of prevailing infrastructure in the country. It offers solutions that are cost effective. It fulfills all the requirements of the security of networks which includes verification, nonrepudiation, integrity, and confidentiality. It is a safe online transaction system with a high potential for receiving the trust of customers and merchants.

Aigbe and Akpojaro[23] offer a comprehensive review of the various categories of electronic payment systems in the context of online payment processes, verification mechanism, and types of authentication. The paper proceeds to prove the application of various verification mechanisms and categories of the electronic payments systems that are emphasized. Eventually, the study disclosesthat electronic payment system with verification mechanisms that involve two or more aspects of authentication are inclined to be safer, with minimized chances of being vulnerable to fraud, and augment the confidence of users in utilizing electronic payment systems. Additionally, this paper requires a combination of the verification mechanisms discussed above, specifically, the three-factor verification model - that includes the biometric (finger-vein) to devise an improved algorithm for electronic payment systems where the capability to verify would exceed the prevalent applications for online payment.

Jesudoss et al. [46]suggested Payment Punishment approach with different models towards inspire the actuality expressive during cluster nodes as well as acknowledge the successful data exchange amongst nodes/clusters. Further, they compared the efficiency of this approach with QoSOLSR protocol. In future, they planned to combine the intrusion avoidance and detection in the occurrence of mischievous nodes. Also, distribute the bandwidth amongst nodes character throughout service delivery.

Kim et al. [24]brought out an effective mutual verification that was based on ID with a crucial agreement protocol by resolving earlier issues; hence it was robust and safe against all identified attacks, specifically in the context of a privilegedinsider attack. They discovered that the suggestion of Islam and Biswas [47] and Qiet al. [48] was bogged down by an issue. Hence they examined areas that considered as contentious and suggested considering our protocol which resolves the issues and provides enhanced security against all recognized attacks. Therefore, the suggested protocol will offer improved security when compared with previous protocols. Additionally, if the study progresses to prove in practical terms the security model, there will arise a possibility to obtain a more significant and worthwhile result.

The present study has reviewed 62 articles, ofwhich 3 were from springer digital library, 17 from IEEE digital library, 12 from Elsevier, 6 from ACM, 7 from the specific journal and international journal and 12 from google scholar (Figure 1). Of these 30 studies that were evaluated the secure mobile payment system, Secure Electronic Transaction protocol, Smart card system, Security, privacy, and encryption based protocol. The pictorial representation of the previous method is discussed in figure 2. From the analysis, most of them have focused towards Security, privacy, and encryption based protocol. 


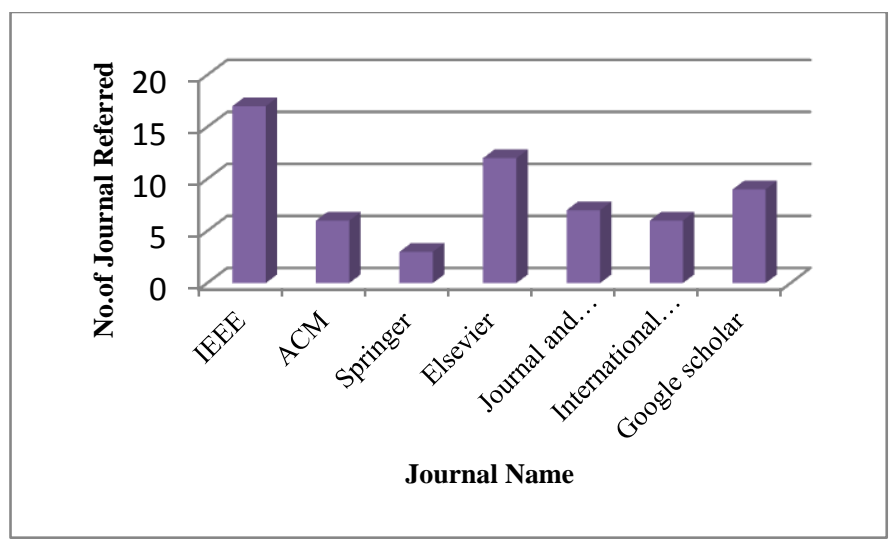

Fig. 1. Pictorial representation of number of research article referred

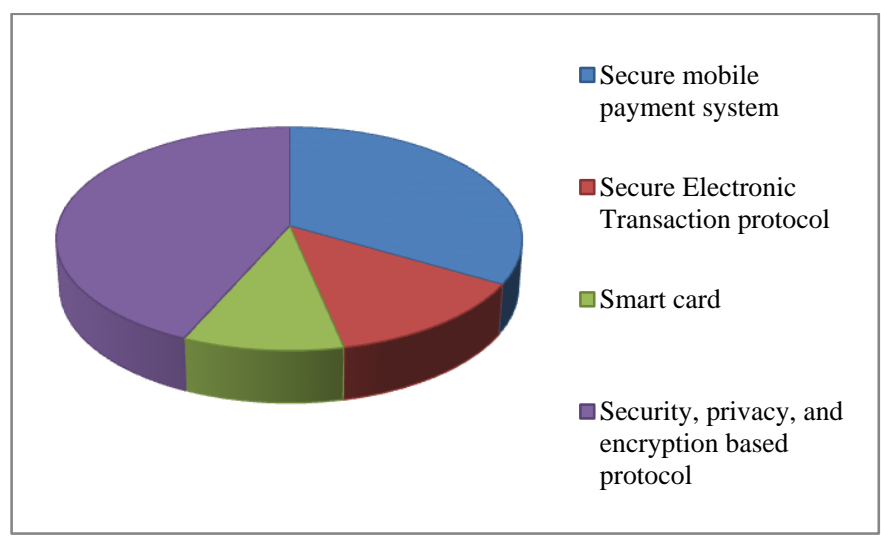

Fig. 2. Pictorial representation of existing method

\section{MATERIALS AND METHODS}

A comprehensive literature search of the secure transaction based online payment system was conducted using a database such as Google search, Elsevier, IEEE, and springer digital library. The data searches were restricted to the period amongst 2004to 2016. From this search retrieved articles included terms associated with secure authentication protocol using mobile payment scheme, smart card, security and privacy based encryption. The search strategies applied are provided in the online supplement. Additionally, these reviewed databases examined the credentials of comprised articles with superiorconcentrations on existing related reviews. As seen in this review, the search retrieved a total of 58 potentially suitable articles to fulfilled inclusion criteria for this review. Here comprised unique investigation studies which were evaluated as well as developed transaction system in e-business, including summarization of the authentication and payment protocol. Further, omitted studies encountered following aspects: (1) Summarization of content outside the ebusiness; (2) merchant and client transaction without epayment; and (3) not written in English might have lost schemes which précis in other languages.

\section{RESULTS AND DISCUSSION}

From the literature, the following problems are identified [49].In Bella et al. [50] the authors proved the dual signature method for payment authorization demand suggests the client on the sender side. In any case, this doesn't promise non- denial. Without a doubt, the investigation did by Herreweghen[51] SET does not convey any protected affirmation to the customer. Likewise in Bella et al. [50] assault against SET is depicted which is like their assault including the nearness of a terrible Payment Gateway, who plots with an awful Merchant to hurt the Cardholder. In Kessler and Neumann [52] and Neumann proposed a confirmation rationale amplifying the rationale AUTLOG utilized for demonstrating the responsibility as a part of the electronic trade and afterward utilize that rationale for checking SET and finished up as secure. Bolignano [53] depicted a confirmation strategy for breaking down the installment conventions by a method for evidence in modular rationale. A contextual analysis has been done on C-SET, a variation of SET. In $\mathrm{Lu}$ and Smolka[54] proposed a disentangled rendition of SET checked with FDR, a model checker taking into account the dialect CSP. Their examination infers that the improved form is secure. However,Panti et al. [55]proposed two assaults on that variant, in spite of the fact that these assaults can't be performed on SET itself. The security of an e-installment technique is essential for all gatherings required in exchange; however, security alone does not ensure accomplishment in the commercial center. An online payment framework should likewise be advantageous. The openness necessity is by and large ignored through security designers whose point is to make the framework as secure as could be expected under the circumstances. Hu et al. [34], Wang and Varadharajan[32], Wang et al. [38], [39], Kim et al. [24], Ismaili et al. [33], Lomte et al. [30], Wu et al. [56] and Ting et al. [37]proposed secure mobile micropayments for e-payment system. However, the performance of security requirements, modules and threads need to be improved by secure authentication and high strength encryption scheme which could be blocked security issues. Also, need to focus towards improving the system performance. Moreover, some protection and security properties were excluded by explored and could be considered and took after as future inquires about Mobahat [43].

On the other hand, some of them focused on lightweight protocol [25], [41]. However, this based on secure payments through online in a limited situation where direct communication amongst customer and mercantile is not possible. Further, the privacy and security properties were not included [57].Previously few of them have focused towards epayment technique, yet just a couple are being utilized effectively. CyberCash, depends on card-based payment[57]. The e-payment not an exactly effective method as credit-card methods [11]. SET is another payment-card based protocol [58]. Further, it not designed as particularly for online payment and secure socket layer [59] based e-payment techniques as extensively used. However, a combination of this technique is possible. Few of them focused on RFID based transceivers authentication. However, the privacy and security properties were not included [43]. Previously, several methods show how to design protocols were future found out to have security breaches [60]. Thus the authentication of secure protocols is key as it can recognize defects which lead towards protocol disappointment. So need to verify this model using online based security protocols. Hu et al. [34],Tab and ehjooy and Nazhand[25] and Isaac and Zeadall[27] they used 
cryptographic method for the transaction. However, the cryptographic-based encryptiondoes not assurance secure operation of the protocol, even if it is a precise method.

Furthermore, few of them have focused smart card-based secure protocol [44], [61], [62]. However, this study needs to improve the proposed solution towards developing a prototype as well as illustrate its efficiency in real time application. Ray et al. [2005] proposed an optimistic protocol in which the trusted third party is invoked only if any party misbehaves or prematurely aborts.However, this study needs to analyze the protocol through formal software specification and verification tools like FDR. In particular, to study the load at the trusted third party and how the frequency failure at third party affects the performance. On the other hand, focused secure payment system using mathematical mode Raghuwanshi et al. [18]. However, this needs to improve the accuracy and efficiency of the calculation. Abdellaoui and Pasquet[19] proposed online payment service provider that accomplishes payment interactions on behalf of the customer and the merchant on the private banking network side. However, this study does not focus on how to integrate three entities (client, merchant,andPSP) in the payment system that involves other parties.

\section{CONCLUSION}

In this study explored the safe authentication based online payment system. From the literature, found no one stated the best way towards secure multicast sessions in e-business operational environment as well as securing e-trade payment for multicast services. To examining the accessible of epayment protocols and limits of their applicability towards online payment system in e-business environment were studied. This protocols and framework are strong substance towards utilizing the unicast e-business platform. In any case, none of this frameworkhas expressed could be applied towards transmitting online payment for multicast e-business environment where the versatility of the foundation will be deliberated as the prime target. To provide the security of client can able to buy the desired items using security methods. This can certify the security of payment system, thus make an incredible solution for e-business by an intermediate in the online payment system. In order to overcome issues of Secure Authentication based e-Payment Protocol, have planned to propose a stylized transaction for online commerce using an intermediary in the e-payment field. This proposed model of intermediary not only settles payments, but it also takes care of such needs as confirming seller and buyer identities, authenticating and verifying ordering and payment information and other transactional requirements lacking in virtual interactions.

- Initially, technical, business and user requirements should be considered for a payment system presenting an interoperable, modular, integrated, and extensible with payment architecture that provides the potentials for deploying security extensions.

- Secondly, according to the specified system requirements, a financial system evaluated and interactions between internal components and external components of the system.
- The third step will identify potentials of the payment model of the system for security enhancement along with preserving system behavior including protocols, services, transactions, and message structure.

- Next, an interface has been designed which is used to interact with the adopted financial system.

- Identifying potentials points of interactions between mobile applications and backbone system in applying security constraints was the starting point to employ security arrangements.

- According to all information related to evaluating system security potentials, security requirement specifications will be determined, so that the system can proceed persistently along with predicted security circumstances.

\section{REFERENCES}

[1] J. Tan, K. Tyler, and A. Manica, "Business-to-business adoption of eCommerce in China,” Inf. Manag., vol. 44, no. 3, pp. 332-351, 2007.

[2] R. Kalakota and A. Whinston, Electronic commerce: a manager's guide. Boston: Addison-Wesley, 1997.

[3] P. M. A. Ribbers and E. V. Heck, "Introducing electronic auction systems in the Dutch flower industry - a comparison of two initiatives," Wirtschaftsinformatik, vol. 4, no. 3, pp. 223-231, 2004.

[4] K. C. Laudon and C. G. Traver, E-commerce: business, technology, society. London: Addison Wesley, 2002.

[5] W. A. Khan, S. Yousaf, N. A. Mian, and Z. Nawaz, "E-commerce in Pakistan: Growth potentials and e-payment solutions," in Proceedings 11th International Conference on Frontiers of Information Technology, FIT 2013, 2013, pp. 247-252.

[6] A. Tiwari, "A Multifactor Security Protocol for Wireless PaymentSecure Web Authentication using Mobile Devices," Indian Institute of Information Technology, Allahabad, 2007.

[7] H. Gupta and V. K. Sharma, "Role of Multiple Encryption in Secure Electronic Transaction,” Int. J. Netw. Secure. Its Appl., vol. 3, no. 6, pp. 89-96, 2011.

[8] Entrust, "White paper : Enhanced Online Banking Security , Zero Touch Multi-Factor Authentication," 2016. .

[9] State Services Commission, Guidance on Multi-factor Authentication. Wellington: State Services Commission, 2006.

[10] D. C. Lynch and L. Lundquist, Digital money: the new era of Internet commerce. Chichester: Wiley, 1996.

[11] P. Wayner, Digital cash: commerce on the Net, 2nd ed. London: AP Professional, 1997.

[12] R. Guttmann, Cybercash: the coming era of electronic money. Basingstoke: Palgrave, 2003.

[13] D. Abrazhevich, Electronic Payment Systems: a User-Centered Perspective and Interaction Design. Eindhoven, The Netherlands: Technische Universiteit Eindhoven, 2004.

[14] I. Ray, I. Ray, and N. Natarajan, "An anonymous and failure resilient fair-exchange e-commerce protocol," Decis. Support Syst., vol. 39, no. 3, pp. 267-292, May 2005.

[15] M. M. Kouta, M. M. Abou Rizka, and A. M. Elmisery, "Secure ePayment using Multi-agent architecture," in Proceedings - International Computer Software and Applications Conference, 2006, vol. 2, pp. 315320.

[16] G. H. Cui, L. Su, M. X. Yang, and Y. Wang, "A secure E-voting system based on list signature for large scale," in First International Conference on Communications and Networking in China, ChinaCom '06, 2007, pp. $1-5$.

[17] A. Tiwari, S. Sanyal, A. Abraham, S. J. Knapskog, and S. Sanyal, "A Multi-Factor Security Protocol for Wireless Payment - Secure Web Authentication using Mobile Devices," in International Conference on 
Applied Computing Proceedings of the IADIS International Conference on Applied Computing, 2007, pp. 160-167.

[18] S. Raghuwanshi, R. K. Pateria, and R. P. Singh, "A new protocol model for verification of payment order information integrity in online Epayment system," in 2009 World Congress on Nature and Biologically Inspired Computing, NABIC 2009 - Proceedings, 2009, pp. 1665-1668.

[19] R. Abdellaoui and M. Pasquet, "Secure communication for internet payment in heterogeneous networks," in Proceedings - International Conference on Advanced Information Networking and Applications, AINA, 2010, pp. 1085-1092.

[20] A. Mazumdar and D. Giri, "On-line Electronic Payment System using signcryption," Procedia Technol., vol. 6, pp. 930-938, 2012.

[21] A. Takyi and P. O. Gyaase, "Enhancing Security of Online Payments: A Conceptual Model for a Robust E-Payment Protocol for E-Commerce," in Contemporary Research on E-business Technology and Strategy: International Conference, iCETS 2012, 2012, pp. 232-239.

[22] O. B. Lawal, A. Ibitola, and O. B. Longe, "Internet Banking Authentication Methods in Nigeria Commercial Banks," African J. Comput. ICT, vol. 6, no. 1, pp. 208-215, 2013.

[23] P. Aigbe and J. Akpojaro, "Analysis of Security Issues in Electronic Payment Systems,” Int. J. Comput. Appl., vol. 108, no. 10, pp. 10-15, 2014.

[24] S. Y. Kim, H. Kim, and D. H. Lee, "An Efficient ID-Based Mutual Authentication Secure against Privileged-Insider Attack," in 2015 5th International Conference on IT Convergence and Security (ICITCS), 2015, pp. 1-4.

[25] A. A. Tabandehjooy and N. Nazhand, "A lightweight and secure protocol for mobile payments via wireless internet in M-commerce," in IC4E 2010 - 2010 International Conference on e-Education, e-Business, e-Management and e-Learning, 2010, pp. 495-498.

[26] A. B. M. R. Sazzad, S. B. Alam, M. N. Sakib, C. Shahnaz, and S. A. Fattah, "Secured cellular banking protocols using virtual internet with digital watermarking," in 2010 IEEE International Symposium on Signal Processing and Information Technology, ISSPIT 2010, 2011, pp. 424427.

[27] J. T. Isaac and S. Zeadally, "An Anonymous Secure Payment Protocol in a Payment Gateway Centric Model," Procedia Comput. Sci., vol. 10, pp. 758-765, 2012.

[28] E. Kim, Y. S. Lee, S. Y. Lee, J. W. Choi, and M. S. Jung, "A study on the information protection modules for secure mobile payments," in 2013 International Conference on IT Convergence and Security, ICITCS 2013, 2013, pp. 1-2.

[29] S. S. Ahamad, V. N. Sastry, and M. Nair, "A Biometric based Secure Mobile Payment Framework," in Proceedings - 4th IEEE International Conference on Computer and Communication Technology, ICCCT 2013, 2013, pp. 239-246.

[30] V. Lomte, S. Deshmukh, S. Jadhav, and V. Munde, "A Secure MPayment Protocol for Mobile Devices," Int. J. Emerg. Res. Manag. \&Technology, vol. 3, no. 4, pp. 75-79, 2014.

[31] S.-U. Guan, S. . Tan, and F. Hua, "A Modularized Electronic Payment System for Agent-based E-commerce," 2004.

[32] Y. Wang and V. Varadharajan, "A Mobile Autonomous Agent-based Secure Payment Protocol Supporting Multiple Payments," in IEEE/WIC/ACM International Conference on Intelligent Agent Technology, 2006, pp. 88-94.

[33] H. El Ismaili, H. Houmani, and H. Madroumi, "A Secure Electronic Transaction Payment Protocol Design and Implementation," Int. J. Adv. Comput. Sci. Appl., vol. 5, no. 5, pp. 172-180, 2014.

[34] Z.-Y. Hu, Y.-W. Liu, X. Hu, and J.-H. Li, "Anonymous micropayments authentication (AMA) in mobile data networks," in IEEE INFOCOM 2004, 2004, vol. 1, pp. 46-53.

[35] H. Suryotrisongko and B. Setiawan, "A Novel Mobile Payment Scheme based on Secure Quick Response Payment with Minimal Infrastructure for Cooperative Enterprise in Developing Countries," Procedia - Soc. Behav. Sci., vol. 65, no. 1, pp. 906-912, 2012.

[36] A. M. Mwafise and L. Stapleton, "Determinants of user adoption of mobile electronic payment systems for microfinance institutions in developing countries: Case study cameroon," IFAC Proc. Vol., vol. 45, no. 10, pp. 38-43, 2012.

[37] H. Ting, Y. Yacob, L. Liew, and W. M. Lau, "Intention to Use Mobile Payment System: A Case of Developing Market by Ethnicity," Procedia - Soc. Behav. Sci., vol. 224, no. 1, pp. 368-375, 2016.

[38] Y. Wang, K.-L. Tan, and J. Ren, "PumaMart: a parallel and autonomous agents based internet marketplace," Electron. Commer. Res. Appl., vol. 3, no. 3, pp. 294-310, Sep. 2004.

[39] Y. Wang, K.-L. Tan, and J. Ren, "A Study of Building Internet Marketplaces on the Basis of Mobile Agents for Parallel Processing," World Wide Web, vol. 5, no. 1, pp. 41-66, 2002.

[40] D. Lange and O. Mitsuru, Programming and Deploying Java Mobile Agents with Aglets. Boston: Addison-Wesley Pub Co, 1998.

[41] A. Sun, "Optimization Study for Lightweight Set Protocol," in 2012 International Conference on Industrial Control and Electronics Engineering, 2012, pp. 1206-1209.

[42] J. Sfenrianto, "A Model of Factors Influencing Consumer's Intention to Use E-payment System in Indonesia," Procedia Comput. Sci., vol. 59, no. 1, pp. 214-220, 2015.

[43] H. Mobahat, "Authentication and lightweight cryptography in low cost RFID," in ICSTE 2010 - 2010 2nd International Conference on Software Technology and Engineering, Proceedings, 2010, vol. 2, pp. V2-123-V2129.

[44] N. El Madhoun, F. Guenane, and G. Pujolle, "A cloud-based secure authentication protocol for contactless-NFC payment," in 2015 IEEE 4th International Conference on Cloud Networking, CloudNet 2015, 2015, pp. $328-330$

[45] D. Pal, V. Vanijja, and B. Papasratorn, "An Empirical Analysis towards the Adoption of NFC Mobile Payment System by the End User," Procedia Comput. Sci., vol. 69, no. 1, pp. 13-25, 2015.

[46] A. Jesudoss, S. V. Kasmir Raja, and A. Sulaiman, "Stimulating truthtelling and cooperation among nodes in VANETs through payment and punishment scheme," Ad Hoc Networks, vol. 24, no. PA, pp. 250-253, 2015.

[47] S. H. Islam and G. P. Biswas, "An improved ID-based client authentication with key agreement protocol on ECC for mobile clientserver environments," Theor. Appl. Informatics, vol. 24, no. 4, p. $293-$ 312, 2012.

[48] Y. Qi, C. Tang, M. Xu, and B. Guo, "An identity-based mutual authentication with key agreement scheme for mobile client-server environment," in Communications Security Conference (CSC 2014), 2014, 2014, pp. 1-5.

[49] S. Brlek, S. Hamadou, and J. Mullins, "A flaw in the electronic commerce protocol SET," Inf. Process. Lett., vol. 97, no. 3, pp. 104108, Feb. 2006.

[50] G. Bella, F. Massacci, and L. Paulson, "The verification of an industrial payment protocol: the SET purchase phase," in Proc. 9th ACM Conf. on Computer and Comm. Security, 2002, pp. 12-20.

[51] E. Van Herreweghen, "Non-repudiation in SET: open issues, in: Proc. 4th Conf. on Financial Cryptography," in Lecture Notes in Computer Science, 2001, vol. 1962, pp. 140-156.

[52] V. Kessler and H. Neumann, "A sound logic for analysing electronic commerce protocols," in 5th European Symposium on Research in Computer Security Louvain-la-Neuve, 1998, pp. 345-360.

[53] D. Bolignano, "Towards the formal verification of electronic commerce protocols," in Proceedings 10th Computer Security Foundations Workshop, 1997, pp. 133-146.

[54] S. Lu and S. A. Smolka, "Model checking the secure electronic transaction (SET) protocol," in MASCOTS '99. Proceedings of the Seventh International Symposium on Modeling, Analysis and Simulation of Computer and Telecommunication Systems, 1999, pp. 358-364.

[55] M. Panti, L. Spalazzi, S. Tacconi, and S. Valenti, "Automatic verification of security in payment protocols for electronic commerce," in Proc. 4th Internat. Conf. on Enterprise Inform. Systems (ICEIS'02), 2002, pp. 968-974. 
[56] J. Wu, C. Liu, and D. Gardner, "A Study of Anonymous Purchasing Based on Mobile Payment System," Procedia Comput. Sci., vol. 83, pp. 685-689, 2016.

[57] A. Levi and C. K. Koc, "CONSEPP: CONvenient and secure electronic payment protocol based on X9.59," in Proceedings - Annual Computer Security Applications Conference, ACSAC, 2001, vol. 2001-Janua, pp. 286-295.

[58] MasterCard, SET Secure Electronic Transaction Specification (Book 1: Business Description). New York (NY): MasterCard Inc., 1997.

[59] A. O. Freier, P. Karlton, and P. C. Kocher, The SSL Protocol Version 3. Mountain View, CA: Netscape Communications Corp., 1996.
[60] S. Muhammad, Z. Furqan, and R. K. Guha, "Understanding the intruder through attacks on cryptographic protocols," in 44th Annual ACM Southeast Conference, ACMSE 2006, 2006, vol. 2006, pp. 667-672.

[61] M. Badra and R. B. Badra, "A Lightweight Security Protocol for NFCbased Mobile Payments," Procedia Comput. Sci., vol. 83, no. 1, pp. 705-711, 2016.

[62] T. T. T. Pham and J. C. Ho, "The effects of product-related, personalrelated factors and attractiveness of alternatives on consumer adoption of NFC-based mobile payments," Technol. Soc., vol. 43, no. 1, pp. 159$172,2015$. 